\title{
Versatile Wearable Computer for Drivers
}

\author{
Gyouhyung Kyung ${ }^{1}$, Songyi Chae ${ }^{1}$, Kyung Hyun Nam ${ }^{1}$, \\ Kyungmin Lee ${ }^{2}$, and Wanjae Shin ${ }^{2}$ \\ ${ }^{1}$ School of Design and Human Engineering, \\ Ulsan National Institute of Science and Technology, Korea \\ ${ }^{2}$ Human Factors \& Devices Research Team, Hyundai-Kia Motors, Korea \\ \{ghkyung, robim0314, skarudgus\} @unist.ac.kr, \\ $\{\mathrm{kmlee}$, downer $2 \mathrm{k}\}$ @hyundai.com
}

\begin{abstract}
A versatile wearable computer for drivers (VWCD) was proposed that can extend in-vehicle multi-modal display spaces. In the first development phase, LEDs, mini video displays, earphones, and vibrators were included as visual, auditory and tactile displays, and were all attached to an eyeglass frame. One electrocardiographic electrode was attached to the VWCD to obtain the driver's heart rate signal at the posterior auricular artery, and a gyro sensor was used to track the driver's head position. Finally, a Bluetooth device was included to enable communication between VWCD and its mobile phone platform.
\end{abstract}

Keywords: multi-modal display, visual display, wearable computer for drivers.

\section{Introduction}

One of the major reasons to use a wearable computer is that it can seamlessly provide information to people on the move. While wearable computers have been used in various application areas including games, medical devices, aerospace, and automotive industry (for maintenance), there are some problems in current wearable computers that hinder their commercial success. In terms of design and human factors aspects, many wearable computers are still obtrusive and uncomfortable due to their bulky and heavy components such as control box and cables. Therefore, size minimization and wireless connection are a key factor for improving its design. In addition, many wearable devices still tend to be expensive and inefficient [1]. Currently, there is no commercialized wearable computer for drivers [2], despite surface vehicles are most commonly used to move people and objects.

Among display types, visual display is most widely used in the vehicle, followed by auditory and tactile displays. Visual information in the vehicle is usually displayed in the area placed in front of the driver (e.g., cluster, crash pad, and windshield), which is however not always included in the driver's forward field of view (e.g., when the driver look at the outside mirror or the passenger on his side). If the driver does not look ahead, it is very likely for him to miss critical information displayed on conventional in-vehicle visual displays. Therefore, it would be very effective to track the driver's head position and provide critical information right in front of the driver's eye. By doing so, the driver would be less likely to miss 
important information, even when the driver does not look at the forward direction of the vehicle.

There are an ever increasing number of drivers who wear eyeglasses, due to the rapidly growing aging driver population with degraded eyesight. Wearable devices which are mounted on, or integrated into, the eyeglasses, hence, seem to a promising solution to provide safety-related information for drivers. VWCD has been conceptualized to continuously provide the driver safety-related information, as well as to monitor driver's health information. By integrating LEDs, speakers and vibrators on the frame of glasses as visual, auditory, and tactile displays, the driver can always be informed of the direction of imminent risky objects and situations, regardless of their head position, unless their eyes are closed. With electrocardiogram sensors which are attached to the leg ends of the eyeglasses, VWCD can record the driver's heart rate at the bilateral posterior auricular arteries.

VWCD has been designed with the aims to minimize its size and weight, and to consider its appearance, and connection with widely used nomadic devices (e.g., iPhone and iPod Touch). It should also improve the driver's situational awareness.

\section{Related Research}

To implement wearable computers, a variety of display technologies have been developed together. There are various types of display technologies such as single crystal silicon, liquid crystal on silicon, high temperature poly silicon, low temperature poly silicon, laser or optical beam-scan, organic light-emitting diode, digital micro-mirror device, and micro-cathode ray tubes. For cost effectiveness and minimization, VWCD uses LEDs and mini video displays to provide visual information to both eyes.

Currently, there are five different methods to display visual information; binocular immersive, binocular see-periphery, binocular see-through (navigation/video sunglasses), monocular see-around, and monocular see-through. As a first phase of developing VWCD, binocular see-around types and mini video display have been selected to provide additional visual information, while minimizing obstruction of

Table 1. Ambient vs. Focal Visual Systems (from [6])

\begin{tabular}{|l|l|l|}
\hline & Ambient System & Focal System \\
\hline Primary Functions & $\begin{array}{l}\text { Visual guidance; motor } \\
\text { control }\end{array}$ & $\begin{array}{l}\text { Form recognition; } \\
\text { identification }\end{array}$ \\
\hline $\begin{array}{l}\text { LGN (Lateral Geniculate } \\
\text { Nucleus) source }\end{array}$ & Magnocellular & Parvocellular \\
\hline Cortical stream & Dorsal stream & Ventral stream \\
\hline Spatial resolution & Low & High \\
\hline Contrast sensitivity & $\begin{array}{l}\text { Asymptotic at low (10\%) } \\
\text { contrast }\end{array}$ & $\begin{array}{l}\text { Requires mid-to-high } \\
\text { contrast }\end{array}$ \\
\hline Spatial frame of reference & $\begin{array}{l}\text { Egocentric (absolute body } \\
\text { coordinates) }\end{array}$ & $\begin{array}{l}\text { Allocentric (relative object } \\
\text { space) }\end{array}$ \\
\hline Temporal resolution & High & Low \\
\hline Primary control mode & Closed-loop & Open-loop \\
\hline Memory requirements & Low & Moderate-highs \\
\hline
\end{tabular}


focal vision [6]. Ambient and focal visual systems have different properties, and hence these differences should be considered when designing a visual display system for drivers.

There are several commercialized eyeglasses-type wearable computers such as a wearable display developed by Konica Minolta, an image overlay system for medical data visualization, the Olympus Mobile Eye-Trek model, and the Personal Display by Eyewear.

\section{VWCD System Development}

\subsection{Innovative System Concept Generation by TRIZ and Lateral Thinking Tools}

In order to provide drivers with context-driven information, collecting appropriate and accurate information from the driver and the vehicle is essential. One of the best ways is to use physiological data such as brain waves, heart rate, and body temperature, while minimizing the driver's distraction. Systems that measure drivers' fatigue (e.g., MIT smart car [3]) have not been successfully commercialized due to intrusiveness of the system.

According to Edward De Bono, the problem solver should explore different ways of examining a challenging task [4]. By adopting his concept, called Lateral Thinking, we can think of a system that can obtain the driver's physiological data as well as provide relevant information to the driver. As a measuring device, an eyeglass-type system appears to be less intrusive than other types of alternatives for drivers (clothes, wristband type, etc), considering that many people already get used to wearing corrective eyeglasses or sun glasses. In addition, VWCD provides drivers with information necessary for safe driving while allowing them to maintain their forward sight. Furthermore, multi-modal (visual, tactile, and auditory) displays enhance information transfer by redundancy gain and provide selective, effective display channels, depending on driving context. The multi-functionality and dimensionality change suggested by TRIZ to develop inventive systems are hence applied to the VWCD concept generation, resulting that it functions as input as well as output device, and extends space for visual and auditory displays and sensors to eyeglasses [5].

\subsection{System Components}

The VWCD system consists of a pair of see-around visual displays, mini video displays, vibrators, earphones, and electrocardiogram sensors, a gyro sensor, an operating system, two control / processing units, and a Bluetooth transceiver. Fourteen LEDs are attached around each lens (Figures 1 and 2). Gyro sensor was used to detect rotation and direction of the driver's head movement. LEDs were controlled by the control unit and when drivers turn their head to the right direction while the potentially risky object is detected from the front or left direction, then the left vibrator turns on and the left LEDs blink to alert the driver. When the driver looks at his right side, with the head rotated, left LEDs and vibrators come into play. LEDs can communicate wirelessly with a laptop via the Bluetooth module. Visual displays and earphones can be connected to a mobile application, iPod Touch, and the 
driver can see the icon such as traffic signals using their peripheral vision and hear the alerting sound. Two electrocardiogram sensors are connected to the processing unit and these continuously record the driver's heart activities.

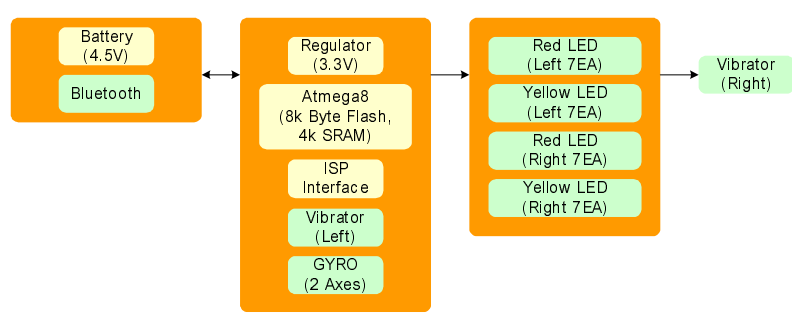

Fig. 1. VWCD block diagram

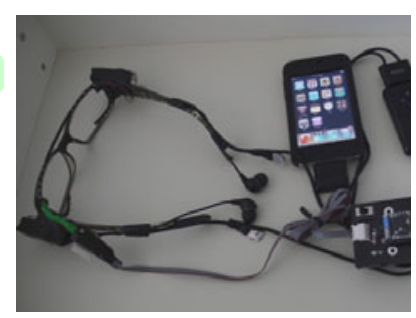

Fig. 2. VWCD prototype

\section{Future Work}

Visually overloaded drivers can easily miss important information. VWCD extends display design space, and facilitates critical information reception by providing multimodal information. However, it should be determined later what combination of input modalities is optimal for the drivers. In the current development phase, a seearound visual display type was selected majorly due to its low cost and functional simplicity, whereas an optical see-through type has not been considered due to its high cost and technological immaturity. In the future, visual displays such as video and optical see-through might be used to more effectively detect the forward driving situation and to display critical information directly on lenses during driving. VWCD always contacts with the driver's skin, which is necessary condition to continuously monitor health-related information. VWCD and its processing unit are wire connected. As a fashionable product integrated with new technologies, VWCD components should be wirelessly connected and miniaturized further to increase users' technology acceptance, and hence, the possibility of commercial success.

\section{References}

1. Rügge, I., Ruthenbeck, C., Reiter, B.S.: Changes of HCI Methods towards the Development Process of Wearable Computing Solutions. In: Kurosu, M. (ed.) HCII 2009. LNCS, vol. 5619, pp. 302-311. Springer, Heidelberg (2009)

2. Randell, C.: Wearable Computing: A Review (2005)

3. Healey, J., Picard, R.: SmartCar: detecting driver stress. In: Proc.15th Int. Conf. Pattern Recognition, Barcelona, Spain, vol. 4, pp. 218-221 (2000)

4. De Bono, E.: New think; the use of lateral thinking in the generation of new ideas. Basic Books, New York (1968)

5. Altshuller, G.: 40 Principles Extended Edition: TRIZ Keys to Technical Innovation, vol. 1 (2005)

6. Castro, C.: Human Factors of Visual and Cognitive Performance in Driving. CRC Press, Boca Raton (2009) 